\section{International Scientific Journal Theoretical \& Applied Science}

\author{
p-ISSN: 2308-4944 (print) e-ISSN: 2409-0085 (online) \\ Year: 2017 Issue: $01 \quad$ Volume: 45 \\ Published: $13.01 .2017 \quad \underline{\text { http://T-Science.org }}$
}

Gennady Evgenievich Markelov Candidate of Engineering Sciences, associate professor, corresponding member of International Academy of Theoretical and Applied Sciences, Bauman Moscow State Technical University, Moscow, Russia markelov@bmstu.ru

SECTION 2. Applied mathematics. Mathematical modeling.

\title{
WORKING MATHEMATICAL MODEL OF ELECTRO-THERMAL SYSTEM COMPONENT
}

Abstract: A mathematical model of a resistor with temperature-dependent conductivity and full heat capacity is obtained within the scope of a unified approach to working mathematical model building. The constructed model has the properties of fullness, accuracy, adequacy, productivity, and economy to a sufficient degree for the purposes of this study. The use of such a mathematical model lowers costs and time spent on studies, and allows expedient use of mathematical modeling capabilities.

Key words: working mathematical model, properties of mathematical models, principles of mathematical modeling.

Language: Russian

Citation: Markelov GE (2017) WORKING MATHEMATICAL MODEL OF ELECTRO-THERMAL SYSTEM COMPONENT. ISJ Theoretical \& Applied Science, 01 (45): 1-3.

Soi: $\underline{\text { http://s-o-i.org/1.1/TAS-01-45-1 Doi: crossef https://dx.doi.org/10.15863/TAS.2017.01.45.1 }}$

РАБОЧАЯ МАТЕМАТИЧЕСКАЯ МОДЕЛЬ ЭЛЕМЕНТА ЭЛЕКТРОТЕПЛОВОЙ СИСТЕМЫ

Аннотация: В рамках единого подхода к построению рабочей математической модели получена математическая модель резистора, проводимость и полная теплоемкость которого зависят от температуры. Построенная модель в достаточной мере обладает свойствами полноты, точности, адекватности, продуктивности и экономичности применительно к данному исследованию. Применение такой математической модели приводит к сокращению затрат времени и средств на проведение исследования, позволяет рачионально использовать возможности математического моделирования.

Ключевые слова: рабочая математическая модель, свойства математических моделей, принципь математического моделирования.

\section{1. Введение}

Подходы к построению математических моделей различных технических систем изложены в обширной учебной и научной литературе. В работах $[1 ; 2]$ введено понятие рабочей математической модели и изложен единый подход к построению математической модели, которая в достаточной мере обладает нужными свойствами применительно к конкретному исследованию. Некоторые свойства математических моделей приведены в работах [3; 4]. В работе [5] рассмотрен пример построения математической модели, в достаточной мере обладающей нужными свойствами применительно к исследованию, некоторые результаты которого изложены в работах [6-8]. Особенности внедрения единого подхода к построению математических моделей рассмотрены в работах $[9 ; 10]$.

Целью настоящей работы является разработка в рамках единого подхода рабочей математической модели резистора, проводимость и полная теплоемкость которого зависят от температуры.

\section{2. Постановка задачи}

Резистор считаем высокотеплопроводным телом, температура $T$ которого в начальный момент времени $t_{0}$ равна $T_{0}$. На поверхности резистора площадью $S$ происходит конвективный теплообмен с окружающей средой, температура которой равна $T_{0}$, коэффициент теплоотдачи известен и равен $\alpha$. Пусть

$$
G(T)=G_{0}\left[1+\beta\left(T-T_{0}\right)\right],
$$




\begin{tabular}{|c|c|c|c|c|c|c|}
\hline Impact Factor: & $\begin{array}{l}\text { ISRA (India) } \\
\text { ISI (Dubai, UAE } \\
\text { GIF (Australia) } \\
\text { JIF }\end{array}$ & $\begin{array}{l}=1.344 \\
=0.829 \\
=0.564 \\
=1.500\end{array}$ & $\begin{array}{l}\text { SIS (USA) } \\
\text { PИНЦ (Russia) } \\
\text { ESJI (KZ) } \\
\text { SJIF (Morocco) }\end{array}$ & $\begin{array}{l}=0.912 \\
=0.234 \\
=1.042 \\
=2.031\end{array}$ & $\begin{array}{l}\text { ICV (Poland) } \\
\text { PIF (India) } \\
\text { IBI (India) }\end{array}$ & $\begin{array}{l}=6.630 \\
=1.940 \\
=4.260\end{array}$ \\
\hline
\end{tabular}

$$
C(T)=C_{0}\left[1+\gamma\left(T-T_{0}\right)\right], \quad T_{0} \leq T \leq T_{1},
$$

где $G(T)$ и $C(T)$ - проводимость и полная теплоемкость резистора; $G_{0}$ и $C_{0}$ проводимость и полная теплоемкость резистора при $\quad T=T_{0} ; \quad \beta \quad$ и $\quad \gamma \quad-$ температурные коэффициенты, причем $\beta>0$ и $\gamma>0$. Разность электрических потенциалов на полюсах рассматриваемого элемента равна

$$
U=\frac{I}{G_{0}\left[1+\beta\left(T-T_{0}\right)\right]},
$$

где $I$ - сила постоянного электрического тока, протекающего через резистор.

Пусть в рамках проводимого исследования представляет интерес разность электрических потенциалов $U$. Построим рабочую математическую модель объекта исследования, которая в достаточной мере обладает свойствами полноты, точности, адекватности, продуктивности и экономичности.

\section{3. Решение}

Для решения поставленной задачи выстроим иерархию математических моделей данного объекта исследования и определим условия, при выполнении которых можно с относительной погрешностью не более заданного значения $\delta_{0}$ найти искомую величину $U$.

Если разность $T-T_{0}$ достаточно мала, то согласно (1) найдем искомую величину по формуле

$$
U_{0}=I / G_{0} .
$$

Определим условия, при которых применима полученная формула. Для этого рассмотрим установившийся процесс теплообмена. В этом случае мощность тепловыделения в материале резистора равна тепловому потоку, отводимому от резистора, т. е.

$$
\frac{I^{2}}{G_{0}\left[1+\beta\left(T_{*}-T_{0}\right)\right]}=\alpha\left(T_{*}-T_{0}\right) S, \quad T_{0} \leq T_{*} \leq T_{1},
$$

где $T_{*}$ - установившееся значение температуры резистора. Из полученного равенства легко найти

$$
T_{*}=T_{0}+\frac{1}{2 \beta}\left(-1+\sqrt{1+\frac{4 \beta I^{2}}{\alpha S G_{0}}}\right),
$$

а затем определить установившееся значение

$$
U_{*}=I / G\left(T_{*}\right)=\frac{2 U_{0}}{1+\sqrt{1+4 \beta I U_{0} /(\alpha S)}}
$$

при выполнении неравенства

$$
T_{*} \leq T_{1},
$$

которое можно записать в следующем виде

$$
\frac{\beta I^{2}}{\alpha S G_{0}} \leq \beta^{2}\left(T_{1}-T_{0}\right)^{2}+\beta\left(T_{1}-T_{0}\right) .
$$

Очевидно, что $U_{*} \leq U \leq U_{0}$. Тогда для относительной погрешности величины $U_{0}$ запишем

$$
\delta\left(U_{0}\right)=\left|\frac{U-U_{0}}{U}\right|=\frac{U_{0}}{U}-1 \leq \frac{U_{0}}{U_{*}}-1 .
$$

Следовательно, при выполнении условия

$$
\frac{U_{0}}{U_{*}}-1 \leq \delta_{0}
$$

можно с относительной погрешностью не более $\delta_{0}$ использовать формулу (2) для нахождения искомой величины. Тогда приходим к неравенству

$$
\frac{\beta I^{2}}{\alpha S G_{0}} \leq \delta_{0}^{2}+\delta_{0} .
$$

При выполнении неравенств (4) и (5) математическая модель (2) в достаточной мере обладает свойствами полноты, точности, адекватности, продуктивности и экономичности.

Определим условия, при которых применима математическая модель (3). Для этого рассмотрим неустановившийся процесс теплообмена. В этом случае изменение температуры резистора во времени $t$ описывает обыкновенное дифференциальное уравнение первого порядка

$$
C(T) \frac{d T}{d t}=\frac{I^{2}}{G(T)}-\alpha\left(T-T_{0}\right) S, \quad T \leq T_{1},
$$

а начальное условие имеет вид

$$
T\left(t_{0}\right)=T_{0} .
$$

Учитывая, что

$$
U=\frac{U_{0}}{1+\beta\left(T-T_{0}\right)},
$$

сформулируем задачу Коши

$$
\begin{aligned}
& \frac{d U}{d t}=\frac{\beta U^{2}\left[\alpha S\left(U_{0}-U\right)-\beta I U^{2}\right]}{C_{0} U_{0}\left[\gamma\left(U_{0}-U\right)+\beta U\right]}, \\
& U\left(t_{0}\right)=U_{0},
\end{aligned}
$$

где $U \geq \frac{U_{0}}{1+\beta\left(T_{1}-T_{0}\right)}$.

При выполнении условия

$$
\delta\left(U_{*}\right)=\left|\frac{U-U_{*}}{U}\right|=1-\frac{U_{*}}{U} \leq \delta_{0}
$$

можно с относительной погрешностью не более $\delta_{0}$ использовать формулу (3) для нахождения искомой величины, причем

$$
\delta_{0}<\frac{U_{0}}{U_{*}}-1,
$$

так как в противном случае следует применять формулу (2). Затем найдем момент времени

$$
t_{*}=t_{0}+\frac{C_{0}}{\alpha S}\left[\frac{\gamma}{\beta}\left(\frac{U_{*}}{U_{0}}-1+\delta_{0}\right) \frac{U_{0}}{U_{*}}+\left(\frac{U_{0}}{2 U_{0}-U_{*}}+\right.\right.
$$




$$
\begin{aligned}
& \left.+\frac{\gamma}{\beta} \frac{U_{0}-U_{*}}{2 U_{0}-U_{*}} \frac{U_{0}}{U_{*}}-1\right) \ln \left(2-\frac{U_{*}}{U_{0}}-\delta_{0}\right)- \\
& \left.-\left(\frac{U_{0}}{2 U_{0}-U_{*}}+\frac{\gamma}{\beta} \frac{U_{0}-U_{*}}{2 U_{0}-U_{*}} \frac{U_{0}}{U_{*}}\right) \ln \left(\frac{U_{0}}{U_{0}-U_{*}} \delta_{0}\right)\right],
\end{aligned}
$$

для которого $U\left(t_{*}\right)=U_{*} /\left(1-\delta_{0}\right)$. Тогда согласно (7) установившееся значение $U_{*}$ можно с относительной погрешностью не более $\delta_{0}$ считать равным $U(t)$ при $t \geq t_{*}$.

Если справедливо неравенство (4), но не выполнено условие (5), то математическая модель (3) при $t \geq t_{*}$ в достаточной мере обладает свойствами полноты, точности, адекватности, продуктивности и экономичности.

\section{4. Результаты}

Построение иерархии математических моделей позволяет выявить рабочую математическую модель, которая в достаточной мере обладает нужными свойствами применительно к конкретному исследованию. Действительно, если выполняются неравенства (4) и (5), то математическую модель (2) считаем рабочей. Если справедливо неравенство (4), не выполнено условие (5), а временной интервал от $t_{0}$ до $t_{*}$ можно в рамках проводимого исследования не рассматривать, то выбираем математическую модель (3) как рабочую, иначе - математическую модель (7).

\section{5. Заключение}

Таким образом, сформулированы утверждения, которые позволяют установить рабочую математическую модель элемента электротепловой системы. Построенная в рамках единого подхода математическая модель в достаточной мере обладает свойствами полноты, точности, адекватности, продуктивности и экономичности применительно к данному исследованию.

Очевидно, что применение такой модели приводит к сокращению затрат времени и средств на проведение исследования, позволяет рационально использовать возможности математического моделирования.

\section{References:}

1. Markelov GE (2015) On Approach to Constructing a Working Mathematical Model. ISJ Theoretical \& Applied Science, 04 (24): 287-290. Soi: http://s-o-i.org/1.1/TAS-04-2452 Doi: http://dx.doi.org/10.15863/TAS.2015.04.24.52

2. Markelov GE (2015) Constructing a Working Mathematical Model. ISJ Theoretical \& Applied Science, 08 (28): 44-46. Soi: http://s-oi.org/1.1/TAS-08-28-6 Doi: http://dx.doi.org/10.15863/TAS.2015.08.28.6

3. Myshkis AD (2014) Elements of the Theory of Mathematical Models [in Russian]. LENAND, Moscow.

4. Zarubin VS (2010) Mathematical Modeling in Engineering [in Russian]. Izd-vo MGTU im. N.E. Baumana, Moscow.

5. Markelov GE (2012) Peculiarities of Construction of Mathematical Models. Inzhenernyi zhurnal: nauka i innovatsii, No. 4, Available:

http://engjournal.ru/catalog/mathmodel/hidden/ 150.html (Accessed: 03.11.2012).

6. Markelov GE (2000) Effect of initial heating of the jet-forming layer of shaped-charge liners on the ultimate elongation of jet elements. J. Appl. Mech. and Tech. Phys., 41, No. 2, pp. 231-234.

7. Markelov GE (2000) Effect of initial heating of shaped charge liners on shaped charge penetration. J. Appl. Mech. and Tech. Phys., 41, No. 5, pp. 788-791.

8. Markelov GE (2000) Influence of heating temperature on the ultimate elongation of shaped-charge jet elements. Proc. of the 5th Int. Conf. "Lavrentyev Readings on Mathematics, Mec hanics and Physics", Lavrentyev Institute of Hydrodynamics, Novosibirsk, pp. 170.

9. Markelov GE (2015) Particular Aspects of Teaching the Fundamentals of Mathematical Modeling. ISJ Theoretical \& Applied Science, 05 (25): 69-72. Soi: http://s-o-i.org/1.1/TAS$\underline{05-25-14}$ Doi: http://dx.doi.org/10.15863/TAS.2015.05.25.14

10. Markelov GE (2016) Teaching the Basics of Mathematical Modeling. Part 2. ISJ Theoretical \& Applied Science, 01 (33): 72-74. Soi: http://s-o-i.org/1.1/TAS-01-33-15 Doi: http://dx.doi.org/10.15863/TAS.2016.01.33.15 